\title{
Polar bear hunt strategies evaluated by a Leslie matrix population model
}

\author{
NILS ARE ØRITSLAND AND RAY SCHWEINSBURG
}

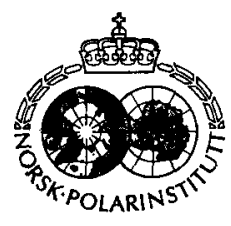

Øritsland, N. A. \& Schweinsburg, R. 1983: Polar bear hunt strategies evaluated by a Leslie matrix population model. Polar Research 1 n.s., 241-247.

An APL program for a Leslie matrix population projection is developed and applied to analysis of a Canadian polar bear population. Hunting specified with respect to sex ratio within a total quota may be included in the projections. Application to the lower central arctic islands' polar bear population of 1100 animals indicates a growth to 1560 animals during ten years without hunting. Projection with two males taken for each female indicates that 54 animals may be taken from the population each year. However, the same sized hunt, but with a one-to-one sex ratio, caused a significant depression of the population.

Nils Are Øritsland, Institute of Zoophysiology, University of Oslo, Box 1051, Blindern, Oslo 3, Norway; Ray Schweinsburg, N.W.T. Wildlife Service, Government of the Northwest Territories, Yellowknife, N.W.T., Canada.

\section{Introduction}

Leslie matrix population models have been available for about 50 years and use information on sex ratio, sex and age specific survivorship, and the age specific productivity or fecundity to predict population development (Caughley 1977). The effect of hunting is rarely included as a separate feature in computer programs executing such models because losses from hunting may be included with natural mortality when considering the survivorship of the population. Field work on polar bears carried out during the last decade has produced demographic values encompassing nearly 2000 individuals. Analysis of available data in the context of matrix models is, however, not yet a routine part of polar bear studies. Thus it is of both general and specific interest for polar bear studies to have computer programs available that offer matrix calculations with the effect of hunting as users' options.

The present work concerns a Leslie matrix population projection model including such a hunt option. The model accepts input fecundity and age structures blindly, i.e. it assumes that the necessary biological considerations regarding density dependencies and age structure stability are already included in the input. On this basis the model is used to evaluate the yield of the polar bear population of the lower central arctic islands of Canada.

\section{Methods}

Three main subroutines are used in the program package: The subroutine LFTAB generates a lifetable from input age frequencies adjusting, of course, the first age class to the value 1 . Also LFTAB will generate the mortality rate of each sex and age class and vectors, named distribution vectors, that are used to distribute the initial lumped population into age classes for each sex.

The optional subroutine HUNT requires a hunt size and strategy in terms of a males/females ratio. The actual matrix calculations projecting the population into the future are performed by the subroutine HTRU.

Below follows a more detailed description of the major events executed by each subroutine.

Within LFTAB the distribution vectors are calculated as follows

$\mathrm{PM}_{\mathrm{i}}=\frac{\mathrm{OFX}_{\mathrm{i}}}{\sum_{\mathrm{i}=1}^{\mathrm{n}} \mathrm{OFX}_{\mathrm{i}}}$

where $\mathrm{i}$ is the age (years) of the males and OFX is the number of males of age $i$ in the population sample. Thus $\mathrm{OFX}_{\mathrm{i}}$ is a vector (string of values) where each element represents the number of males in that sample age class.

The same type of formula is used for females. LFTAB will accept as input two separate sex specific age frequencies or one frequency common 
for both sexes. In the case of a common frequency a 1 to 1 sex ratio is used.

If the input age frequencies, i.e. population samples, are sex specific, LFTAB will generate a corresponding sex ratio which is applied to the initial total population prior to PM and PF. The mortality rates of males and females are generated by LFTAB in the conventional way of any lifetable (see Caughley 1977) and will be used by the matrix subroutine HTRU unless other rates are specified after execution of LFTAB.

As indicated earlier, the routine HUNT will prompt the user to set a sex ratio for the hunt, for example two males for each female killed. For the present application to a Canadian subpopulation HUNT includes the practical experience that the two first age classes are not hunted (Schweinsburg et al. 1980) and accepts the finding that the age distribution of the hunted animals is equal to the distribution of the live population sample. The routine will distribute the hunt of each sex according to the initial input age frequencies for animals older than two years. Thus the hunt option of the model is only a modification of equation 1 and, continuing with the males as an example, the killed animals are distributed into a vector HPM according to:

$$
\mathrm{HPM}_{i=3.4 \ldots \pi}=\frac{\mathrm{OFX}_{i=3.4 \ldots \pi}}{\sum_{i=3}^{n} \mathrm{OFX}_{i}}
$$

Next the actual numbers of hunted animals of each age class are determined by multiplying the total number of animals killed with the appropriate distribution vector, e.g. males:

$$
\mathrm{HM}_{\mathrm{i}=3,4 \ldots \mathrm{n}}=\mathrm{HPM}_{\mathrm{i}=3.4 \ldots \mathrm{n}} \cdot \mathrm{HNM}
$$

where HNM is the total number of males killed by hunters and

$$
\mathrm{HNM}=\mathrm{H}-\mathrm{HNF}
$$

where $\mathrm{H}$ is the total number of animals hunted and HNF is the total number of females hunted.

The totals of males (HNM) hunted are determined by multiplying the grand total of the hunt $(\mathrm{H})$ with integer fractions given for total number of females:

$$
\mathrm{HNF}=\mathbf{H} \cdot \frac{\mathrm{HFF}}{\mathrm{HFF}+\mathrm{HMM}}
$$

where HFF is an integer ratio value of females (for example 1) and HMM is the integer ratio values of males (for example 2 ) in the harvest.
Thus if the sex ratio of the polar bear kill is, as indicated by Schweinsburg et al. 1980, two males to each female, equation 5 becomes $\mathrm{HNF}=\mathrm{H} / 3$. Obviously then $\mathrm{HNM}=2 / 3 \mathrm{H}$.

In HTRU, the Leslie matrix population projection subroutine, the initial population size is distributed into sex specific age frequencies by multiplying the population size $(\mathrm{P})$ with the distribution vectors, e.g. males:

$\mathrm{M}_{\mathrm{i}=1,2 \ldots \mathrm{n}}=\mathrm{P} \cdot \mathrm{PM}_{\mathrm{i}=1.2 \ldots \mathrm{n}}$

where $\mathrm{PM}_{\mathrm{i}}$ is the distribution vector (eq. 1).

Next the age distribution of the hunted animals are subtracted:

$M_{i=1.2 \ldots n}=M_{i=1,2 \ldots n}-H_{i=3,4 \ldots n}$

A winter mortality is subsequently applied thus reducing the population by its mortality in one step before the breeding season. Still using the males as example:

$\mathbf{M}_{\mathrm{i}}=\mathrm{M}_{\mathrm{i}}-\left(\mathrm{M}_{\mathrm{i}} \cdot \mathrm{MRM}_{\mathrm{i}}\right)$

where MRM is the age specific mortalities. New individuals are added to the population by multiplying the female age frequency vector $F$ by its age specific productivity (PR) and adding the products:

$\mathrm{C}=\sum_{i=1}^{m} \mathrm{~F} \cdot \mathrm{PR}$

where $m$ is the maximum age of the females. The newborn animals may be subject to an optional early mortality (MRCS) and a 1:1 sex ratio for the surviving newborn is assumed.

Subsequently the newborn are added as the youngest age classes of the population at the same time as the oldest age classes are erased. A time step, i.e. a year, is counted, and the sequence of events starting with subtraction of hunted animals is repeated. An abbreviated flow diagram of the sequence of events is illustrated in Fig. 1.

The model is written in APL as documented by the program listings (appendix).

\section{Results}

For the lower central arctic islands of Canada a polar bear subpopulation of 1100 animals with the common age frequency for both sexes of 88.94 85.2081 .4777 .737470 .1766 .4462 .758 .9755 .23

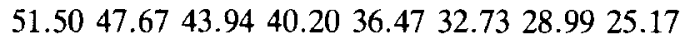




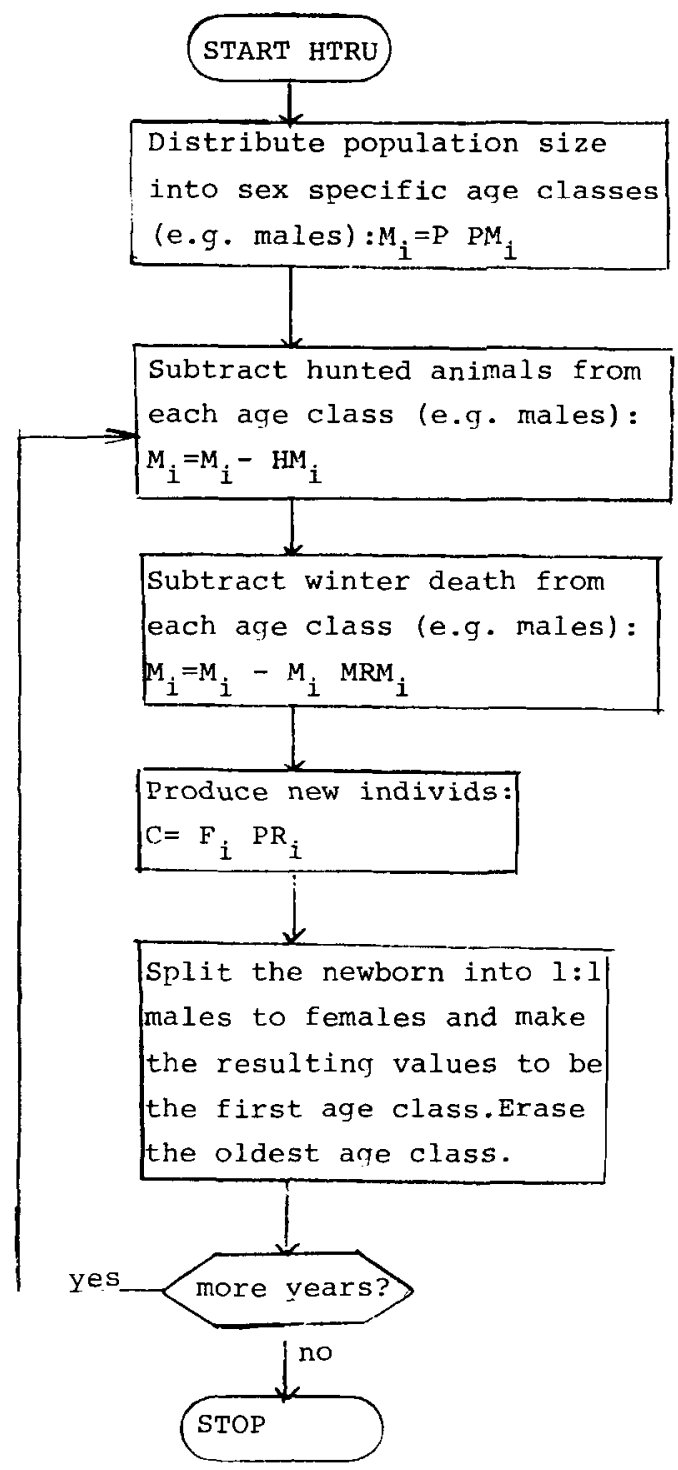

Fig. 1. Abbreviated flow diagram for execution of the subroutine HTRU.

21.4517 .7013 .9610 .236 .492 .67 and an even productivity rate of $0.388 \mathrm{cubs} / \mathrm{female} / \mathrm{year}$ for females older than 3 years has been reported (Schweinsburg et al. 1980).

Projecting the above population without hunting resulted in a population of 1559 animals after 10 years (Fig. 2).

In reality the sex ratio of the hunt is two males to each female (Schweinsburg et al. 1980) which results in a sustainable yield of 54 animals per year from the population of 1100 animals. How-

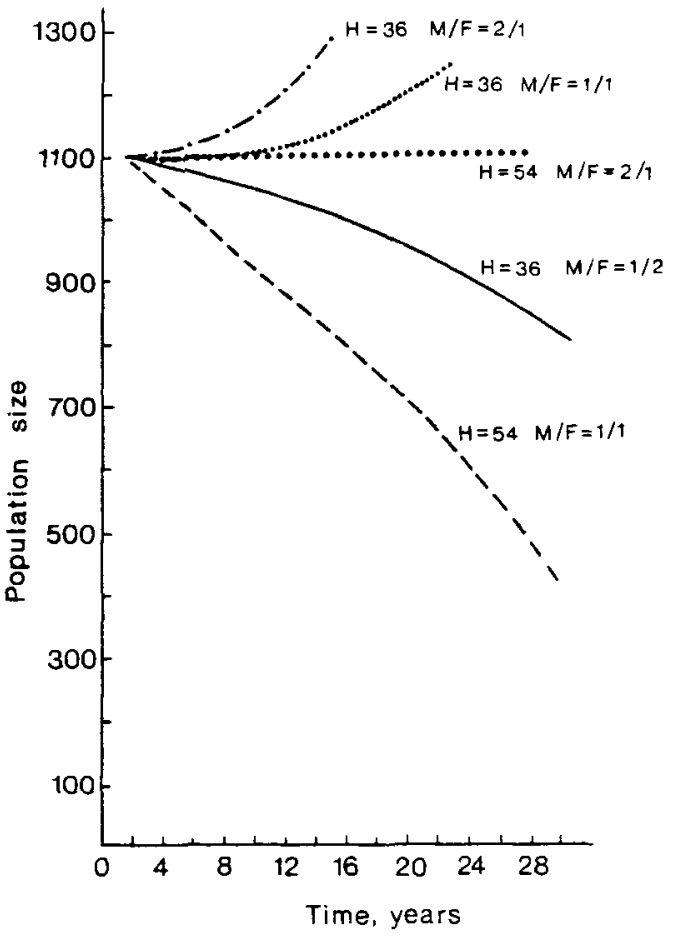

Fig. 2. Prognosis for the lower central Canadian Arctic islands" polar bear population for varying hunt strategies. $\mathrm{H}$ is the total number of animals killed each year and $\mathrm{M} / \mathrm{F}$ is the ratio of males to females in the hunt $(\mathrm{H})$.

ever, the same sized hunt, but with a one to one sex ratio caused a significant depression of the population (Fig. 2).

\section{Discussion}

The use of a stable age structure for hunted animals independent of both hunting quotas and years of extrapolation may be unrealistic. Normally a hunted or predated population will be expected to shift its age frequencies towards younger age classes (Caughley 1977). Thus the present model should perhaps be used only for evaluating the instantaneous yields and concurrent with collection of age and productivity data from the population. In other words, although the present initial age frequencies of both the hunted animals and the living population are in accord with field observations, the model may become less realistic as the population size changes from the present level because of possible density dependent effects not included here. 
There is a paucity of information regarding density related changes in productivity and natural mortality for polar bears. Any hunt provides an opportunity to collect such information. It may be that a regime with controlled variations in hunt quotas and sex ratio provides the most economical and efficient way of collecting such data.

The present work demonstrates the importance of sex ratio considerations in a hunting regime (Fig. 2). A hunt of two males per female (54 animals/year) resulted in a stable population and predicted 877 males and 1001 females after 50 years.

The same quota, however, hunted at one male per female depressed the population. Collection of life statistic parameters is clearly essential to sound management policy.

Acknowledgements. - This work was supported by the Norwegian MAB-programme, the Svalbard project. We thank $J$. Lee. N. Tyler, J. Lentfer, and K. I. Ugland for their comments on the manuscript.

\section{References}

Caughley. G. 1977: Analysis of Vertebrate Populations. J. Wiley. New York. 234 pp.

Schweinsburg, R. Furnell, D. J. \& Miller, S. J. 1980: Abundance. distribution and population structure of polar bears in the lower central arctic islands. N.W.T. Wildlife Service Report. Yellowknife Northwest Territories. 


\section{Appendix 1}

LFTAB Will from the original age frequency data generate the life table vectors $\mathrm{LX}, \mathrm{DX}$ and $\mathrm{QX}$ for both sexes. The values may be displayed by use of TABLE.

$\nabla L F T A B[\square] \nabla$

$\nabla L F T A B$

[1] 'OUTPUT VARS. FOR THIS PROGRAM $A R E$ '

[2] ' $L X, D X$ AND $Q X$ FOR MALES AND FEMALES. IN ORDER TO START'

[3] 'YOU MUST ENTER YOUR ORIG. AGE FREQUENCIES AS'

[4] ' $O F X \leftarrow N N N$ '

[5] 'HAS A VARIABLE NAMED OFX BEEN DEFINED? (1 FOR YES'

[6] '0 FOR NO)'

[7] $A \leftarrow \square$

[8] $\rightarrow(A=0) / G$

[9] 'WAS THAT MALES (M), FEMALES (F) OR BOTH $(B)$ ?'

[10] $A 2 \leftarrow \square$

[11] $\rightarrow\left(\left(A 2={ }^{\prime} M^{\prime}\right), \quad\left(A 2=F^{\prime}\right), \quad\left(A 2={ }^{\prime} B^{\prime}\right)\right) /$ $(L 1, L 2, L 3)$

[12] ACALCULATION OF MALE DISTRIBUTION (PM) AND MORTALITY (MRM) VECTORS

[13] L1: OFXM OFX

[14] 'NOW GIVE FEMALE AGE FREQUENCIES'

[15] $O F X F \leftarrow \square$

[16] $L 7: P M \leftarrow O F X M \div(+/ O F X M)$

[17] $L X M \leftarrow O F X M \div O F X M[1]$

[18] $F X M \leftarrow O F X M((1 \downarrow O F X M), 0)$

[19] $D X M \leftarrow F X M \div(+/ F X M)$

[20] $M R M \leftarrow Q X M \leftarrow D X M \div L X M$

[21] AMFR IS MALE FRACTION

[22] $M F R \leftarrow+/(O F X M+O F X F)$

[23] $M F R \leftarrow(+/ O F X M) \div M F R$

[24] 'MALE VECTORS FINISHED'

[25]

[27] $\rightarrow\left(A 2={ }^{\prime} M '\right) / L 5$

[28] $\rightarrow L 4$

[29] A CALCULATION OF FEMALE DISTRIBUTION (PF) AND MORTALITY (MRF) VECTORS

[30] $L 2: O F X F \leftarrow O F X$

[31] 'NOW GIVE MALE AGE FREQUENCIES'

[32] $O F X F \leftarrow \square$

[33] $L 5: F F R \leftarrow+/(O F X F+O F X M)$

[34] $F F R \leftarrow(+/ O F X F) \div F F R$
[35] $P F \leftarrow O F X F \div(+/ O F X F)$

[36] $L X F \leftarrow O F X F \div O F X F[1]$

[37] $F X F \leftarrow O F X F-((1 \downarrow O F X F), O)$

[38] $D X F \leftarrow F X F \div(+/ F X F)$

[39] $M R F \leftarrow Q X F \leftarrow D X F \div L X F$

[40] 'FEMALE VECTORS FINISHED'

[41] ' '

[42]

[43] $\rightarrow\left(A 2={ }^{\prime} F^{\prime}\right) / L 7$

[44] $\rightarrow L 4$

[45] L3: '50/50 SEX RATIO IS ASSUMED'

[46] $M F R \leftarrow F F R \leftarrow 0.5$

[47] $O F X \leftarrow 0.5 \times O F X$

[48] $P M \leftarrow P F \leftarrow 0.5 \times(O F X \div(+/ O F X))$

[49] $L X M \leftarrow L X F \leftarrow O F X \div O F X[1]$

[50] $F X \leftarrow O F X-((1 \downarrow O F X), O)$

[51] $D X M \leftarrow D X F \leftarrow F X \div(+/ F X)$

[52] $M R M \leftarrow M R F \leftarrow Q X M \leftarrow Q X F \leftarrow$ $D X M \div L X M$

[53] L4: 'LIFETABLE AVAILABLE UNDER 'TABLE".'

[54] $\rightarrow 0$

[55] G: 'ENTER AGE FREQUENCIES IN OFX AND REEXECUTE LFTAB' $\nabla$

TABLE Formats and prints the life table values with four decimals.

\section{$\nabla T A B L E[\square] \nabla$}

$\nabla T A B L E$

[1] $D A T A \leftarrow L X M, L X F, D X M, D X F, Q X M$, $Q X F$

[2] $L L \leftarrow 6,(\rho L X M)$

[3] DATA $\quad L L \rho D A T A$

[4] $D A T A \leftarrow \varnothing D A T A$

[5] ' $L X M L X F D X M D X F Q X M Q X F$ '

[6] 4 Ф DATA $\nabla$

TRU Reminds the user that a) if the demographic variables are to be reset the subroutine (function) LFTAB must be used, b) the productivity of the females is stored in the vector (variable) named $P R$.

Asks the user how many years that are to be projected or calculated for.

Asks for the initial population size.

Asks whether a hunt is to be included in to the calculations.

$\nabla T R U[\square]$

$\nabla T R U$

[1] 'IF DEMOGRAPHIC VARS. ARE TO BE SET:'

[2] 'CHECK THE “LFTAB” PROGRAMME' 

[4] '(REMEMBER THAT PRODUCTIVITY IS IN "PR")'
$[5]$
[6] 'SIMULATE - HOW MANY YEARS?'
[7] $\quad N \leftarrow \square$
[8] 'INITIAL POPULATION SIZE?'
[9] $P \leftarrow \square$
[10] 'HUNT? (ANSWER 1 FOR YES, 0 FOR $N O)^{\prime}$
[11] $H A \leftarrow \square$
[12] $\rightarrow(H A=0) / T R$
[13] $H U N T$
[14] TR:TRUU
$\nabla$

HUNT In case there is to be a hunt during each year of population projection this subroutine will distribute the hunted animals into ageclasses excluding the first years of life $\left(0^{-}\right.$and $\left.1^{\circ}\right)$. HUNT allows the user to define the male to female ratio of the kill.

\section{$\nabla H U N T[\square] \nabla$}

$\nabla H U N T$

[1] 'GIVE TOTAL HUNT'

[2] $\quad H \leftarrow \square$

[3] 'GIVE MALE TO FEMALE RATIO OF THE KILL'

[4] '(USE TWO ENTRIES):'

[5] 'MALES?'

[6] $H M M \leftarrow \square$

[7] 'FEMALES?'

[8] $H F F \leftarrow \square$

[9] $\quad H N M \leftarrow H-H N F \leftarrow$ $H \times(H F F \div(H F F+H M M))$

[10] $H O F X M \leftarrow 2 \downarrow O F X M$

[11] $H O F X F \leftarrow 2 \downarrow O F X F$

[12] $H P M \leftarrow H O F X M \div(+/ H O F X M)$

[13] $H P F \leftarrow H O F X F \div(+/ H O F X F)$

[14] $H F \leftarrow 00,(H N F \times H P F)$

[15] $H M \leftarrow 00,(H N M \times H P M)$

[16] HTRU

TRUU TRUU will as HTRU perform the actual population projection. but does not include the subtraction routine included in line [5] and [7] of HTRU.

\section{$\nabla T R U U[\square] \nabla$ \\ $\nabla T R U U$}

[1] $\quad T I \leftarrow 0$

[2] $M \leftarrow P \times P M$

[3] $F \leftarrow P \times P F$

[4] $P N \leftarrow+/(F+M)$

[5] $C H E: M \leftarrow M-(M \times M R M)$
[6] $\quad F \leftarrow F-(F \times M R F)$

[7] $C \leftarrow+/(P R \times F)$

[8] $C \leftarrow C-(C \times M R C S)$

[9] $C F \leftarrow C M \leftarrow 0.5 \times C$

[10] $F \leftarrow C F, F[\iota(\rho F)-1]$

[11] $M \leftarrow C M, M[\iota(\rho M)-1]$

[12] $N Y \leftarrow(+/ F)++/ M$

[13] $P N \leftarrow P N, N Y$

[14] $T I \leftarrow T I+1$

[15] $\rightarrow(T I<N) / C H E$

[16] $N$; 'YEARS GAVE'; \NY; 'ANIMALS'

[17] 'MALES: '; $\lfloor(+M)$

[18] 'FEMALES: '; $\lfloor(+F)$ $\nabla$

HTRU Performs the actual population projection with subtraction of the animals hunted each year.

$910 \nabla H T R U[\square] \nabla$ $\nabla H T R U$

[1] $T I \leftarrow O$

[2] $M \leftarrow P \times P M$

[3] $F \leftarrow F \times P F$

[4] $P N \leftarrow+/(F+M)$

[5] $C H E: M \leftarrow M-H M$

[6] $M \leftarrow M-(M \times M R M)$

[7] $\quad F \leftarrow F-H F$

[8] $\quad F \leftarrow F-(F \times M R F)$

[9] $C \leftarrow+/(P R \times F)$

[10] $C \leftarrow C-(C \times M R C S)$

[11] $C F \leftarrow C M \leftarrow 0.5 \times C$

[12] $F \leftarrow C F, F[\iota(\rho F)-1]$

[13] $M \leftarrow C M, M[\iota(\rho M)-1]$

[14] $N Y \leftarrow(+/ F)++/ M$

[15] $P N \leftarrow P N, N Y$

[16] $T I \leftarrow T I+1$

[17] $\rightarrow(T I<N) / C H E$

[18] $N$; 'YEARS WITH'; 'HUNTED GAVE'; $\lfloor N Y$; 'ANIMALS ALIVE'

[19] 'MALES: '; $\lfloor(+/ M)$

[20] 'FEMALES: '; $\lfloor(+/ F)$

[21] $\rightarrow 0$

LN Will calculate the rate of increase $(r)$ for the total population according to the formula $P_{t}=P_{0} e^{r t}$ where $P_{0}$ is the initial population and $P_{t}$ is the population size after $t$ years.

$$
\nabla L N[\square]
$$$$
\nabla L N
$$

[1] 'GIVE THE POPULATION SIZE AT START'

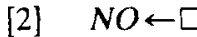

[3] 'GIVE THE POPULATION SIZE AFTER T YEARS' 


\section{[4] $\quad N T \leftarrow \square$ \\ [5] 'HOW MANY YEARS?' \\ [6] $\quad T \leftarrow \square$ \\ [7] $R L N \leftarrow(*(N T \div N O)) \div T$ \\ [8] $R L N$}

\section{List of variables and definitions}

C - total number of newborn (scalar)

$\mathrm{CF}$ - total number of newborn females (scalar)

$\mathrm{CM}$ - total number of newborn males (scalar)

F - age structure for females (vector)

FFR - female fraction of the initial total population (scalar)

HF - the age structure of hunted females (vector)

HM - the age structure of hunted males (vector)

M - the age structure i.e. size of age classes for males (vector)
MFR - male fraction of the initial total population (scalar)

MRCS - the early (post natal) mortality rate of the newborn (scalar)

MRM - vector age specific mortality rates for males (scalar)

$\mathrm{N}$ - number of years to be simulated (scalar)

OFX - input vector where each element represents the age class size of a population sample.

OFX is later declared to be male, female or common for both sexes and thus transformed to OFXM and OFXF

$P \quad$ - the total population size (scalar)

$\mathrm{PF}-$ (distribution) vector where each element is the relative number of females for each class

PM - as for PF, but now concerning males

PN - vector where each element is the total population size for each year of projection

PR - vector where each element is the (age specific) productivity rate of the females 
